Document downloaded from:

http://hdl.handle.net/10251/156845

This paper must be cited as:

Gregori Verdú, S.; Tur Valiente, M.; Tarancón Caro, JE.; Fuenmayor Fernández, F. (2019). Stochastic Monte Carlo simulations of the pantograph-catenary dynamic interaction to allow for uncertainties introduced during catenary installation. Vehicle System Dynamics. 57(4):471-492. https://doi.org/10.1080/00423114.2018.1473617

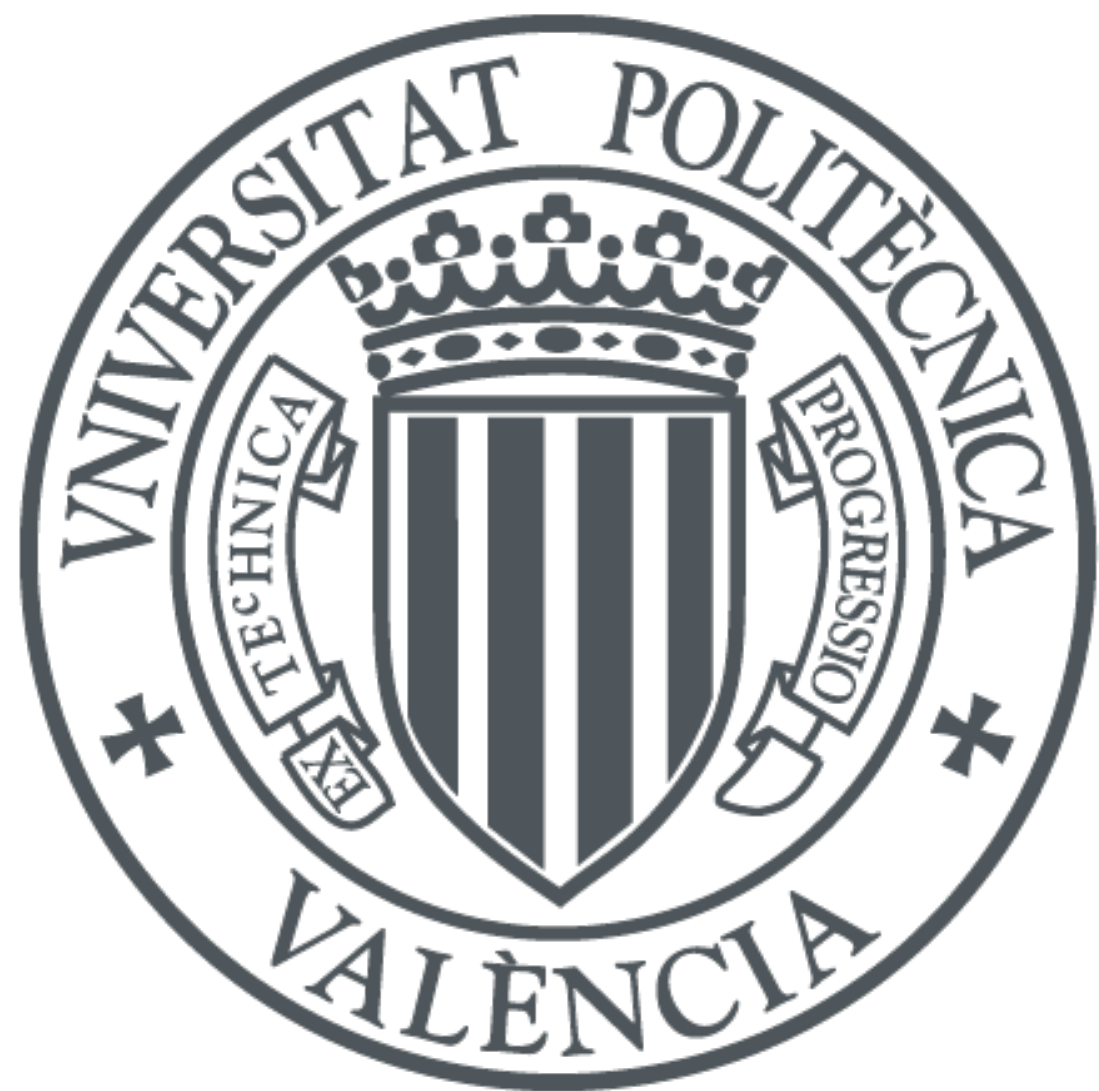

The final publication is available at

https://doi.org/10.1080/00423114.2018.1473617

Copyright Taylor \& Francis

Additional Information

"This is an Accepted Manuscript of an article published by Taylor \& Francis inVehicle System Dynamics on APR 3 2019, available online:

https://www.tandfonline.com/doi/full/10.1080/00423114.2018.1473617." 


\title{
Stochastic Monte Carlo simulations of the pantograph-catenary dynamic interaction to allow for uncertainties introduced during catenary installation
}

\author{
S. Gregori ${ }^{a}$, M. Tur ${ }^{a}$, J.E. Tarancón ${ }^{a}$, F.J. Fuenmayor ${ }^{a}$ \\ ${ }^{a}$ Centro de Investigación en Ingeniería Mecánica \\ Universitat Politècnica de València \\ Camino de Vera s/n, 46022 Valencia, Spain
}

April 23, 2018

\begin{abstract}
The simulation of the pantograph-catenary dynamic interaction is at present mainly based on deterministic approaches. However, any errors made during the catenary stringing process are sources of variability that can affect the dynamic performance of the system. In this paper we analyse the influence of dropper length, dropper spacing and support height errors on the current collection quality by applying a classic Monte Carlo method to obtain the probability density functions of several output quantities. The effects of installation errors are also studied for a range of train speeds. Finally, the pre-sag that, on average, produces the best behaviour of the system is identified, allowing for the uncertainty in the catenary installation. The results obtained show the convenience to consider variability in pantograph-catenary dynamic simulations.
\end{abstract}




\section{Introduction}

Current collection in high-speed trains is achieved by means of a sliding contact between the pantograph and a conductive wire. The former is mounted on the locomotive, whilst the overhead line, the catenary, is a cable structure suspended above the track. According to the standards, the interaction force generated by this sliding contact can be used to determine the quality and stability of current supply. Ideally, this force should have low force peaks to prevent component damage and wear, but at the same time it should be far enough from null values to avoid the undesirable consequences of arcing.

The simulation of the pantograph-catenary dynamic interaction has become an important tool for catenary designers in recent years (see [1] and the references therein). Simulations are helpful when different geometries, configurations and materials need to be tested or even optimized $[2,3]$, with no need for a prototype or expensive in-line tests. However, these simulations usually provide deterministic results, such as optimized geometries or sensitivity studies [4], in which magnitudes like the interaction force or the uplift at a certain point of the catenary do not allow for the variability present in the system.

There are countless sources of variability in the pantograph-catenary dynamic interaction; for example, during catenary stringing some human errors are unavoidably made, leading to a final catenary configuration that differs from the original design. These discrepancies can have severe effects on the dynamic performance of the system, although they are not usually considered in the current software. To the authors' knowledge, very few studies can be found in the literature on the uncertainties of the catenary system. In [5] a diagnosis procedure is proposed to identify catenary defects, such as those produced by creep phenomena, wear in cables and dropper length deviations, whose impact on the catenary dynamic performance are studied in [6]. Guideline values for certain installation error rates based on real measured data were proposed in [7], while variability in pantograph-catenary dynamic simulations regarding wear, aerodynamic effects and geometry irregularities is introduced in [8], in which the need for further studies on the field is suggested.

This paper proposes an approach to deal with the variability introduced by installation errors. Dropper lengths, dropper spacing and support height errors are taken into account 
due to their important role in determining contact wire height [9]. The procedure is based on the application of the well-known classic version of Monte Carlo Simulations (MCS) for the propagation of uncertainty [10] and consists of evaluating the model in a sufficiently large number of simulations with a random sampling of the input quantities as described by their probability density functions (PDFs). A large number of trials are achievable by combining both a highly efficient simulation strategy [11] and parallel computing, so that the effects of mounting errors on the system dynamics are fully characterized in a statistical sense.

The paper is organized as follows: after this brief introduction to the subject, the catenary system and its modelling are described in Section 2. The pantograph-catenary dynamic interaction problem is formulated in Section 3. We use an efficient solution method [11], which provides considerable computational cost savings (a brief description of this method is also presented for the sake of completeness). The installation errors considered and their measurable effects are defined in Section 4. Section 5 is devoted to briefly explaining the Monte Carlo simulation strategy, in which the number of simulated trials is a key factor; this issue is addressed in detail in Section 5.1. Finally, different numerical examples and results are discussed in detail and the conclusions are given in Section 6.

\section{Catenary description and modelling}

The railway catenary is a cable structure through which the power is supplied to the locomotive via a sliding contact with the pantograph. As shown in Fig. 1, the cabling is regularly supported by poles and brackets. In order to keep the contact wire at the desired height it is hung on droppers from the messenger wire. With the help of steady arms, the contact wire is staggered to ensure uniform wear on the pantograph collector strips. Some catenary topologies also include stitch wires near the supports to smooth out stiffness variations.

Although mathematical models and simulations of the pantograph-catenary dynamic interaction were introduced some decades ago, it was not until the appearance of demanding high-speed requirements that an effort was made to improve these models and simulations (see for example $[12,13]$ ). From the huge variety of studies in the recent literature, the 


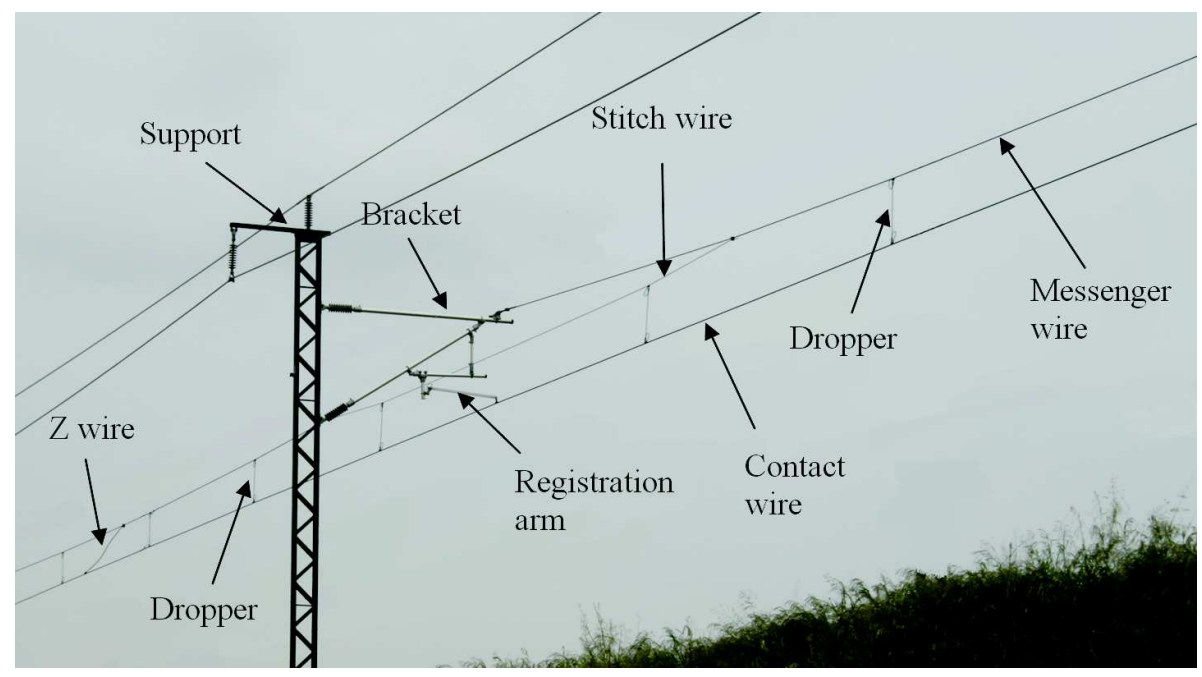

Figure 1: Real catenary with its main components.

benchmark [1] and the references therein stand out from the rest.

To model the catenary we here use the Finite Element model presented in [14], which is based on the Absolute Nodal Coordinate Formulation (ANCF) [15]. All the calculations and examples given in this paper are focused on the Benchmark catenary model, which is depicted in Fig. 2. Its geometric inputs and material properties are fully described in [1].

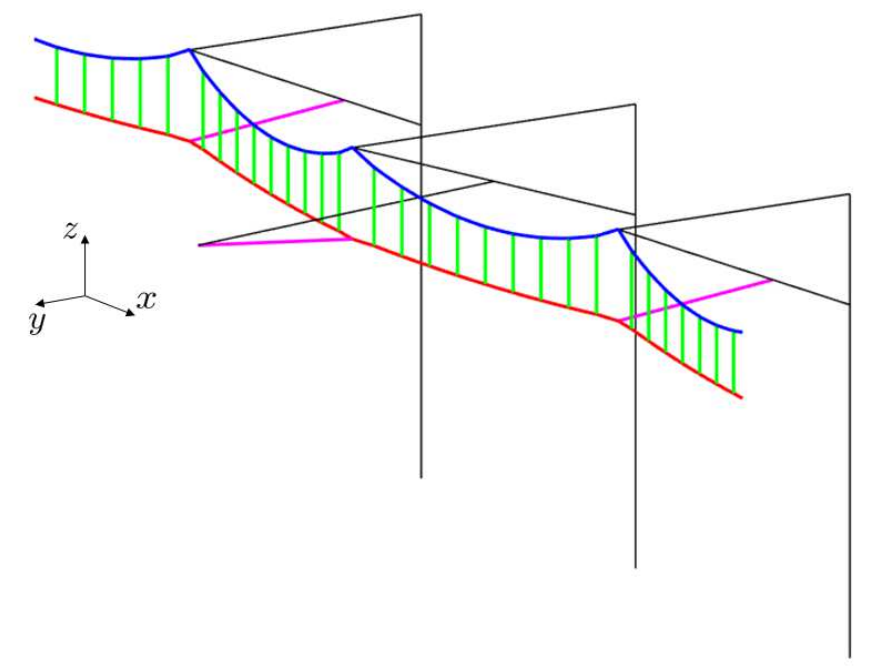

Figure 2: FE model of the benchmark catenary.

Although more complex pantograph models can be found in the literature [16], we use 


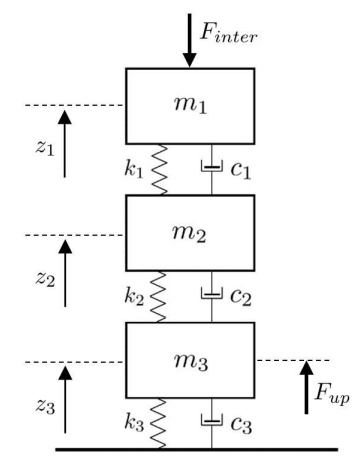

(a)

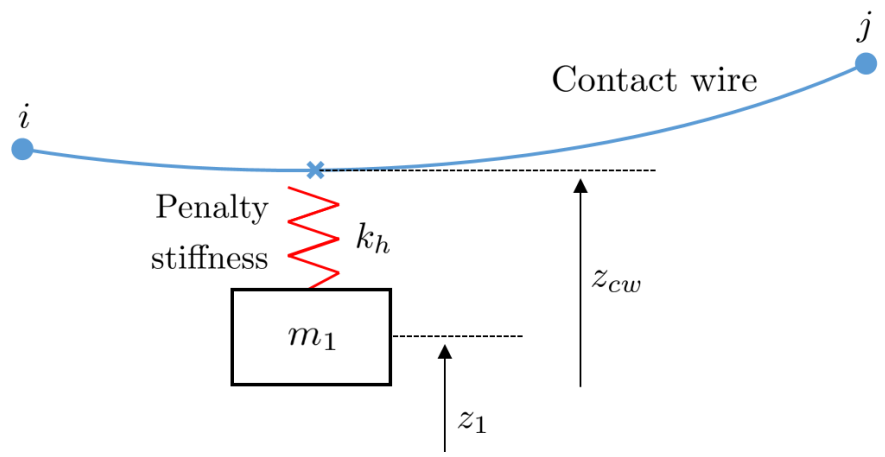

(b)

Figure 3: (a) Pantograph and (b) interaction model schemes.

here a simple lumped-parameter model which only introduces three vertical degrees of freedom. Both pantograph and catenary models interact with each other by means of a penalty method with a high penalty stiffness, $k_{h}=50 \mathrm{kN} / \mathrm{m}$, as recommended in [17]. Fig. 3 contains a sketch of both models.

\section{Dynamic interaction problem}

The static configuration problem consists of calculating the nodal coordinates, $\mathbf{q}$, which fulfil the nonlinear static equilibrium equations (1), that is, the internal forces must balance the gravitational forces:

$$
\mathbf{F}_{\text {int }}(\mathbf{q})+\mathbf{F}_{g}=\mathbf{0}
$$

Nevertheless, in a cabling structure such as the railway catenary, the usual problem to be dealt with is the so-called 'form-finding' problem, in which not only the nodal coordinates $\mathbf{q}$ are unknown, but also the finite element lengths, $\mathbf{l}_{r e f}^{e}$, must be found to fulfil both equilibrium equations and any constraints imposed during the stringing process. Following the procedure proposed in [18] we get:

$$
\begin{aligned}
\mathbf{F}_{i n t}\left(\mathbf{q}, \mathbf{l}_{r e f}^{e}\right)+\mathbf{F}_{g}\left(\mathbf{l}_{r e f}^{e}\right) & =\mathbf{0} \\
\mathbf{c}\left(\mathbf{q}, \mathbf{l}_{r e f}^{e}\right) & =\mathbf{0}
\end{aligned}
$$

The constraint equations $\mathbf{c}$ impose contact and messenger wire tensions, contact wire height and position of droppers and support elements. 
Once the initial configuration is found, dynamic equations can be linearised with respect to this configuration due to the small displacements undergone by the cables. Therefore, if $\mathbf{u}$ denotes for the nodal displacements vector,

$$
\mathbf{M u}+\mathbf{C} \dot{\mathbf{u}}+\mathbf{K u}=\mathbf{F}
$$

models the global pantograph-catenary dynamic interaction problem, in which $\mathbf{M}$ and $\mathbf{K}$ are the mass and stiffness matrices respectively. In this paper, a proportional Rayleigh damping $\left(\alpha_{r}=0.0125\right.$ and $\left.\beta_{r}=10^{-4}\right)$ is considered to build $\mathbf{C}$ [1]. Vector $\mathbf{F}$ contains the external uplift force, $F_{u p}$, applied to the bottom mass of the pantograph model (see Fig. 3a). This dynamic problem is subject to two sources of severe nonlinearities, that is, the unilateral behaviour of droppers and the contact loss between the pantograph and the contact cable.

Although the nonlinear problem stated in Eq. (3) can be solved by the commonly used Newmark method [19], its direct application would require an excessive computational cost for directly using MCS. In order to speed-up the calculations and make the approach viable, in this work we use the Offline/Online strategy proposed in [11]. The solution given by this method is just as accurate as that obtained with the classical direct integration approach. The method is based on two main concepts:

- All sources of nonlinearity are shifted to the right hand side of Eq. (3). These are the interaction force and the compensating force terms coming from the slackened droppers. The idea was first proposed in [13] and exploited in [20]. This fact gives a constant system matrix which is only factorized once in the whole procedure.

- With dynamic responses under unitary forces precomputed in the Offline stage and the application of the superposition principle, the only unknowns are now the value of the correction forces of slackened droppers and the interaction force value at each time step. Thus, one has only to deal with a very small nonlinear system of equations which is iteratively solved during the so called Online stage.

The only disadvantage of this method against the traditional direct integration technique is the need for enough available RAM memory to avoid swapping data onto the hard disk, 
which would slowdown the calculations. For further implementation details the reader is again referred to [11].

\section{Description of catenary installation errors and their effects}

During the catenary installation, technicians can make small mistakes which lead to a different static configuration from the original design. Regarding their role in achieving the desired contact wire height, of the many possible errors we consider here the three deviations from the nominal values given in Fig. 4. Namely:

1) Deviations in dropper length.

2) Deviations in dropper spacing.

3) Deviations in support height.

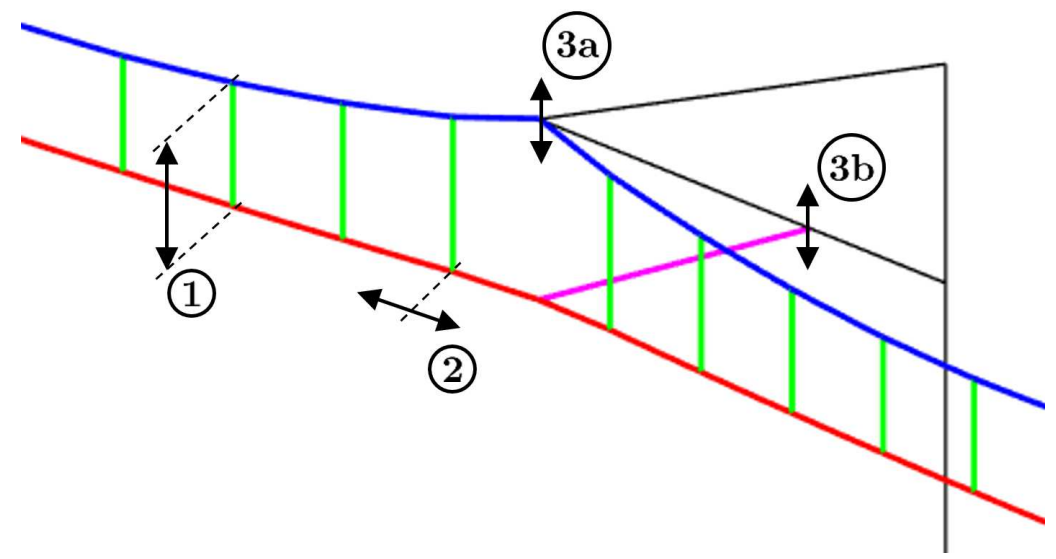

Figure 4: Scheme of installation errors.

In order to quantify these, due to the lack of experimental measurements, we assume they are independent and follow a normal statistical distribution without any loss of generality. The mean is set as $\mu_{i}=0$, for $i=1,2,3$, because each error is defined as the deviation from its design value. The remainder of this section is devoted to providing further details on how each error is considered in the model plus a definition of the measurable magnitudes which can be affected by these errors. 


\subsection{Dropper length error}

The accuracy of dropper measurements during manufacture is reported to be within about $1 \mathrm{~cm}$ [9]. We assume that each dropper length error is a normally distributed variable with standard deviation $\sigma_{1}=6.6 \mathrm{~mm}$, which is in accordance with the value given in [21]. Following the $3 \sigma$ rule, $99.87 \%$ of the length deviations found in droppers will be within $\pm 2 \mathrm{~cm}$.

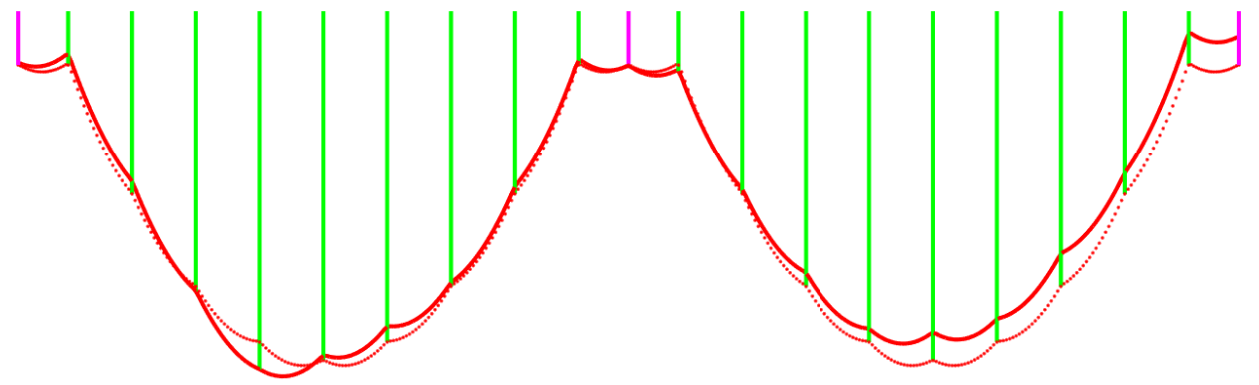

Figure 5: Catenary configuration when including dropper length error (solid line). Nominal configuration (dotted line).

From the computational point of view, in order to model this error we first solve the form-finding problem (2) to obtain the nominal contact wire height. Once the nominal lengths of the dropper elements, $\mathbf{l}_{\text {ref }}^{d}$, are obtained, they are modified by adding to them a value randomly sampled from the normal distribution defined for this type of installation error. The nonlinear static equilibrium problem (1) is then solved with the new dropper lengths to obtain the actual catenary configuration.

Changes in the contact wire profile can be seen in Fig. 5, which shows an example of two spans of the Benchmark catenary with and without dropper length deviations. If we define the span slope, $s_{s}$, as the difference in height per unit length between two consecutive steady arms, it is also slightly affected, mainly by deviations in the length of the droppers near the supports. 


\subsection{Dropper spacing error}

In a $55 \mathrm{~m}$ long span all the droppers are likely to be clamped to the wires at a short distance away from the point planned in the design. In the absence of experimental measurements and available information in the literature, we assume a normal distributed error with standard deviation $\sigma_{2}=20 \mathrm{~mm}$. Again, following the $3 \sigma$ criterion, a deviation of within $\pm 60 \mathrm{~mm}$ with respect to their nominal position will be found in $99.87 \%$ of cases.

During installation, the dropper is initially cut to the nominal length $l_{\text {ref }}^{d}$, which guarantees the contact wire will be at the desired height. However, when the dropper is being clamped to the wires, if it is incorrectly placed, the contact wire height will not be as planned. In order to simulate the realistic features of this error, the following steps are performed:

1. Solve the form-finding problem (2) for the nominal dropper spacing in order to obtain the nominal dropper lengths $\mathbf{l}_{r e f 1}^{d}$ (dashed line in Fig. 6).

2. Solve a new form-finding problem (2) for a catenary with random deviations $\Delta$ in dropper spacing (dotted line in Fig. 6). For this obtained configuration, the obtained dropper lengths $\mathbf{l}_{r e f 2}^{d}$ differ from the nominal ones, $\mathbf{l}_{r e f 1}^{d}$.

3. Restore the nominal lengths $\mathbf{l}_{r e f 1}^{d}$ to the dropper elements and solve a static equilibrium problem (1) to obtain the final catenary configuration (solid line in Fig. 6), which has both nominal dropper lengths and a random dropper spacing error.

\subsection{Support height error}

The last error to be considered is the height at which cantilevers are fixed to the posts. These heights were measured in [21] and show a standard deviation of $\sigma_{3}=2 \mathrm{~cm}$. Sticking to the $3 \sigma$ rule, $99.87 \%$ of the installed catenaries will not have a maximum deviation greater than $6 \mathrm{~cm}$.

To model a catenary with this installation error, the form-finding problem (2) for the nominal catenary must first be solved, followed by the nonlinear static equilibrium problem (1). In the latter, the Dirichlet conditions in $z$ direction of the support nodes of the 


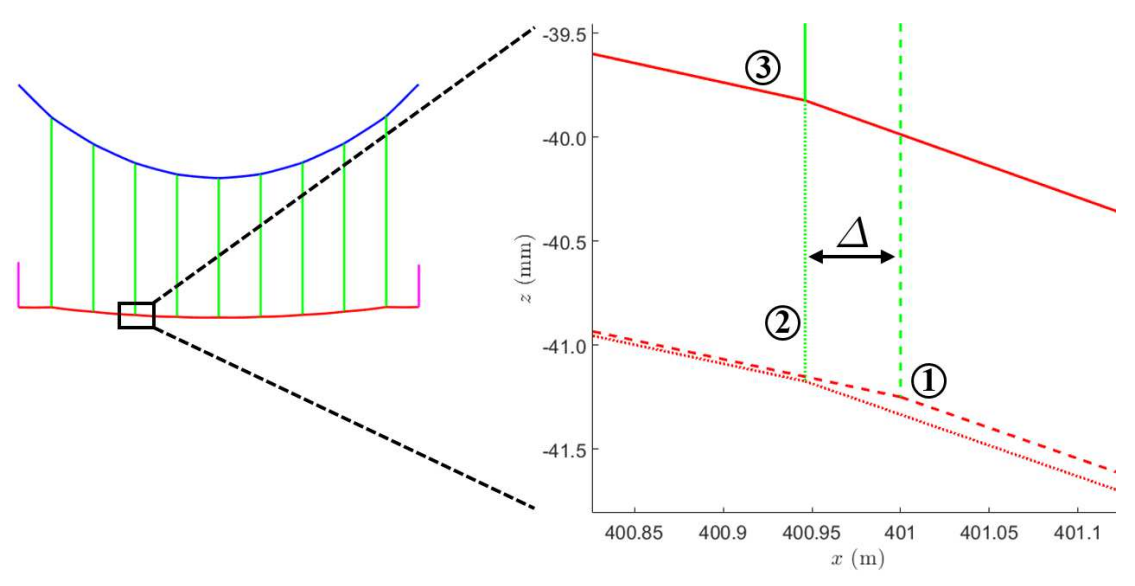

Figure 6: Representation of the catenary configurations obtained after each of the three steps followed to simulate the dropper spacing error.

messenger wire and the end nodes of the steady arms (3a and 3b in Fig. 4) are randomly changed to allow for the support heights error. The random change in height can be either the same or different for both connections of each cantilever, depending on whether they are considered as a single installation error or as two independent deviations.

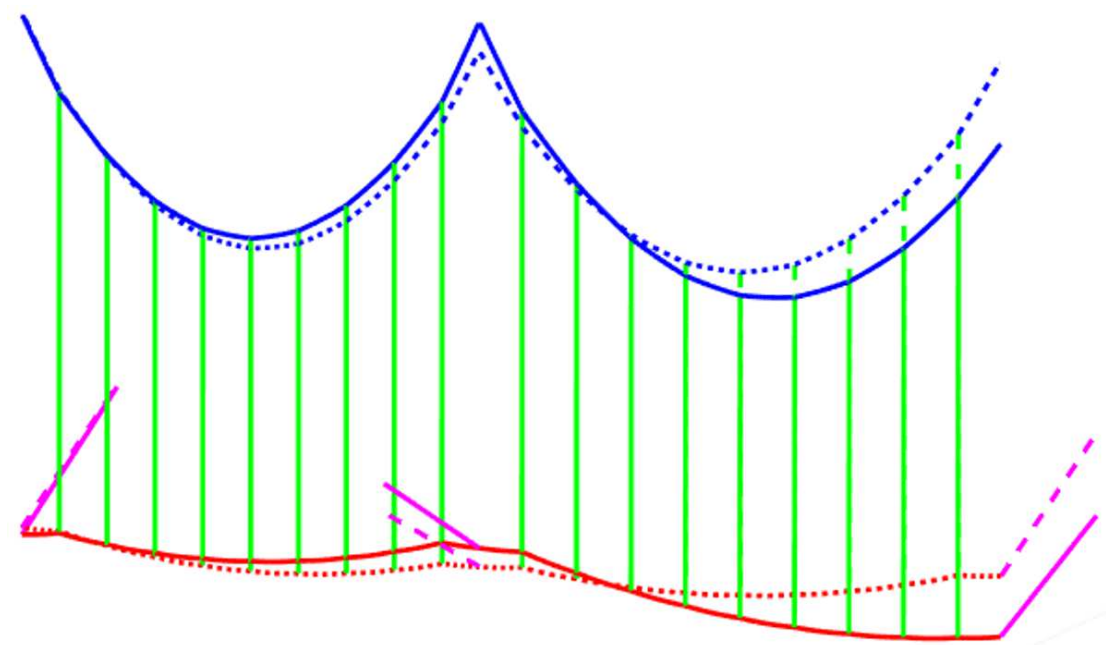

Figure 7: Catenary configuration with deviations in support heights (solid line). Nominal configuration (dashed line).

\subsection{Measurable effects of installation errors}

The installation errors described above affect both the catenary's static configuration and its dynamic behaviour and therefore modify the current collection performance. However, 
measurable magnitudes need to be defined to quantify these effects. In this study we focus on the following parameters, recommended by many standards [22,23] and due to their important role in maintenance procedures:

- Coefficient of variation of the interaction force: defined as the ratio between its standard deviation $\sigma\left(F_{\text {inter }}\right)$, and its mean $\mu\left(F_{\text {inter }}\right)$, namely:

$$
\nu=\frac{\sigma\left(F_{\text {inter }}(t)\right)}{\mu\left(F_{\text {inter }}(t)\right)}
$$

The interaction force is low-pass filtered to $20 \mathrm{~Hz}$ and measured in the ten central spans of a catenary with twenty spans.

- Percentage of time in which contact loss occurs: $t_{c l}$.

- Maximum uplift registered in steady arms: $\Delta z^{\max }$.

- Maximum value of the $20 \mathrm{~Hz}$ filtered interaction force: $F_{\text {inter }}^{\max }$.

- Maximum absolute value of the span slope: $s_{s}^{\max }$.

- Maximum absolute value of the span slope difference between two consecutive spans: $\Delta s_{s}^{\max }$.

The most important magnitude to be evaluated is the ratio $\nu$, by which the current collection quality is mainly characterized. The lower the $\nu$ the more uniform the contact force. However, it is also important to avoid both contact losses and excessive wear on the components, so that $t_{c l}$ and $F_{\text {inter }}^{\text {max }}$ must also be checked. In order to prevent collisions between the pantograph and registration arms, $\Delta z^{\max }$ is usually restricted to 10 or $12 \mathrm{~cm}$. The static parameters $s_{s}^{\max }$ and $\Delta s_{s}^{\max }$ can also be regarded as interesting output quantities due to they are limited by many standards.

\section{Monte Carlo simulations}

The aim of this work was to study the consequences of the errors made during the catenary installation. The widely accepted Monte Carlo (MC) technique was adopted to deal with 
this uncertainty propagation problem [24]. The MC method has certain advantages, such as taking the nonlinearities of the model into account and not imposing any restrictions on the output probability distributions. However, as thousands of dynamic simulations are required this method entails a high computational cost, to alleviate which we resorted to the efficient integration algorithm proposed in [11].

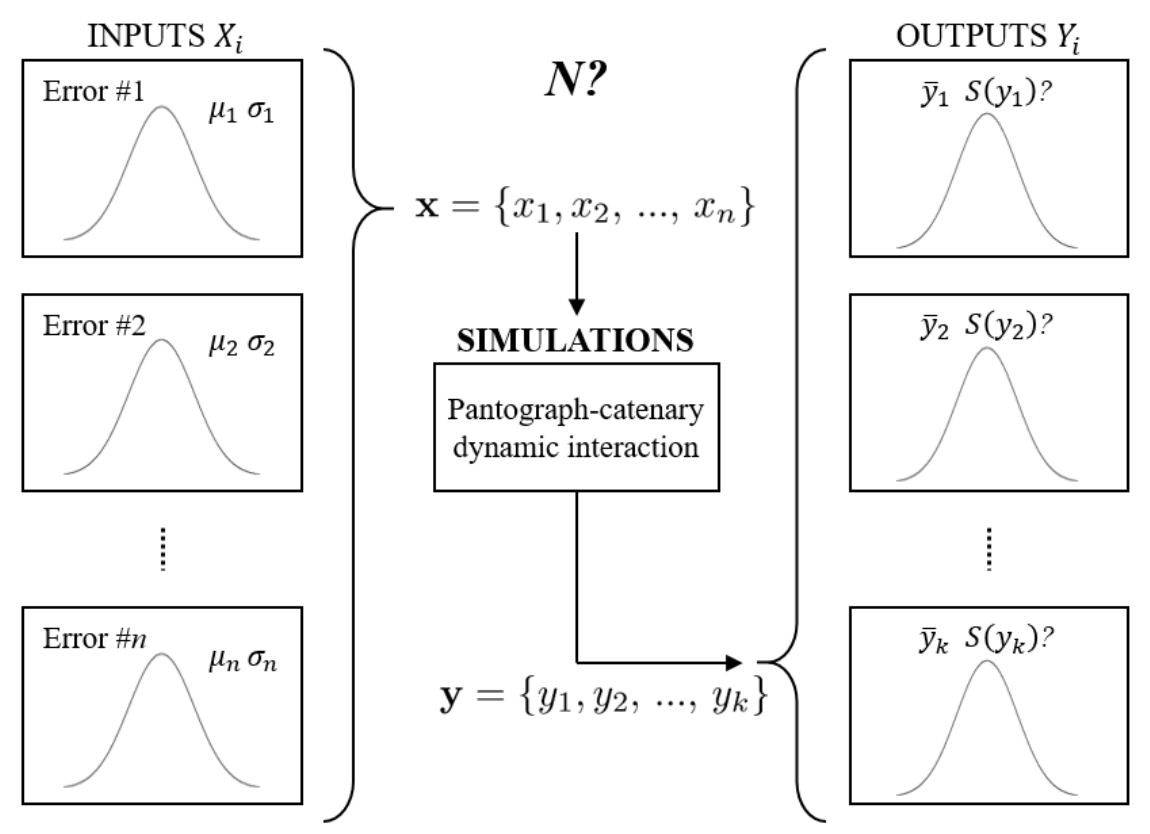

Figure 8: Uncertainty propagation flow diagram.

As can be seen in the diagram shown in Fig. 8, the starting point is the characterization of the statistical distributions $X_{i}$ of the input variables (in this case installation errors). As mentioned in Section 4, they are assumed to be independent Gaussian distributed variables, and are therefore defined by their null mean and standard deviation $\sigma_{i}$. Vectors $\mathbf{x}_{r}, r=1, \ldots, N$, are randomly sampled from the probability density functions and serve as input values for the evaluation of the pantograph-catenary dynamic interaction model.

The key issue in this procedure is how to determine the number of trials, $N$, so that the obtained distributions of the output variables $Y_{i}$, are statistically representative. If we assume that $N$ is chosen to provide enough accuracy in the results, one can build the probability density function (PDF) of each output variable, which can be numerically defined by its statistical moments or coverage intervals. 


\subsection{Searching for the required number of trials}

If $\nu$ is chosen as the output quantity of interest (due to being seen as a good indicator of current collection performance), we need to obtain the value of $N$ which provides enough accuracy in estimating both its real mean $\mu(\nu)$ and its real standard deviation $\sigma(\nu)$. In what follows, these two statistics will be referred to as 'target quantities'.

One simple strategy to asses and control the desired accuracy consists of performing $M$ sets of $N$ simulations, that is, $M$ sets of $N$ catenaries with installation errors, and then measuring the dispersion of the target quantity obtained from each of the $M$ sets. This technique is exemplified in Fig. 9, in which ten sets are considered, $M=10$, for three different trial sizes $(N=500,1000,2000)$. The $M \cdot N$ simulations were performed with a catenary allowing for both dropper length and support height errors, which are the most influential as will be seen later.

Fig. 9 shows the estimated mean $\bar{\nu}$ (left) and standard deviation $S(\nu)$ (right) obtained from each set. Fig. 9 shows that no matter how many trials are performed, the mean

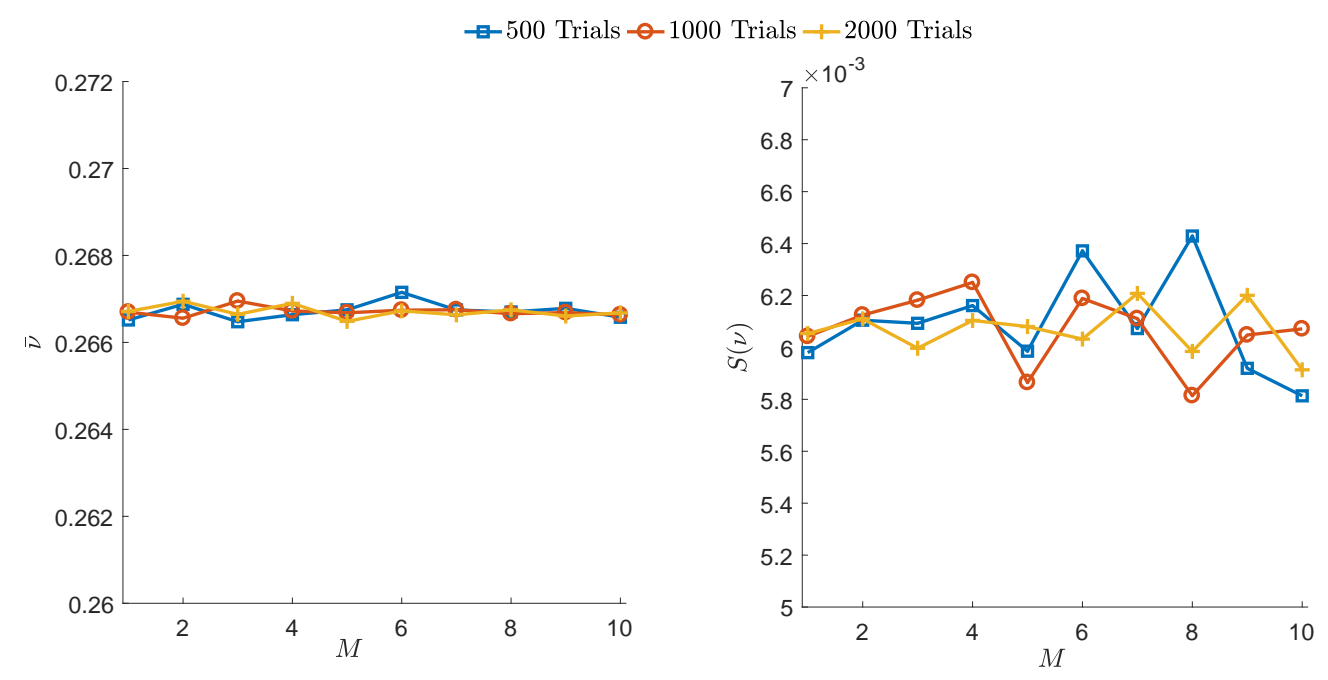

Figure 9: Mean $\bar{\nu}$ (left), and standard deviation $S(\nu)$ (right), for ten sets with different number of trials.

value of $\nu$ barely varies from one set to another, while the standard deviation changes considerably among the different sets, even for the largest ones, $(N=2000)$. However, these results are only able to confirm whether $N$ provides the target quantities with a given 
accuracy when a huge amount of sets $M$ are computed at a prohibitive computational cost.

In order to solve this issue we explore two different approaches. The first strategy is based on the theoretical calculation of the confidence interval of the target quantities. Regarding the unknown population mean $\mu(\nu)$, the central limit theorem [25] establishes that the mean of a sufficiently large sample, $\bar{\nu}$, follows a normal distribution with mean $\mu(\nu)$ and standard deviation $\frac{\sigma(\nu)}{\sqrt{N}}$. If $\alpha$ denotes the significance level, the $100(1-\alpha) \%$ confidence interval of $\mu(\nu)$ is:

$$
\bar{\nu}-z_{\alpha / 2} \frac{\sigma(\nu)}{\sqrt{N}} \leq \mu(\nu) \leq \bar{\nu}+z_{\alpha / 2} \frac{\sigma(\nu)}{\sqrt{N}}
$$

where $z_{\alpha / 2}$ is the value with an occurrence probability lower than $\alpha / 2$ in a normal standard distribution and $N$ is the sample size. We define $d$ as the assumable difference between the sample mean and the unknown population mean, $d=|\bar{\nu}-\mu(\nu)|$, therefore from Eq. (5) we have:

$$
N=z_{\alpha / 2}^{2} \frac{\sigma(\nu)^{2}}{d^{2}}
$$

Although this equation needs the exact value of $\sigma(\nu)$, which is unknown, we can estimate it from a large enough population $(N=5000)$. For the catenaries studied the value of $\sigma(\nu)$ is around 0.265 . Thus, if a $3 \%$ relative error is assumable in the mean estimation, the absolute difference between the estimated and real mean is approximately $d=0.008$. After applying Eq. (6) with a significance level of $\alpha=0.01$, in $99 \%$ of cases $\mu(\nu)$ can be estimated by only four simulations with an error of less than $3 \%$.

Regarding $S(\nu)$, the common approach to obtain the required number of trials $N$ to guarantee a given accuracy of the estimated standard deviation is restricted to normally distributed samples. In our case, as the PDF of $\nu$ is unknown, this normality assumption cannot be made. In this situation, different authors (see for example [26]) propose diverse strategies which only require a finite population kurtosis $\gamma(\nu)$. Among these proposals, a general and simple central limit approach [27] states that the sample variance $S(\nu)^{2}$ is asymptotically normally distributed with an expected value $\sigma(\nu)^{2}$ and a standard deviation $\sigma(\nu)^{2} \sqrt{\frac{\gamma(\nu)-1}{N}}$. This leads to the following nominal $100(1-\alpha) \%$ confidence interval 
for $\sigma(\nu)^{2}$ :

$$
\frac{S(\nu)^{2}}{1-z_{\alpha / 2} \sqrt{\frac{\gamma(\nu)-1}{N}}} \leq \sigma(\nu)^{2} \leq \frac{S(\nu)^{2}}{1+z_{\alpha / 2} \sqrt{\frac{\gamma(\nu)-1}{N}}}
$$

In order to fulfil the last relation for an assumable relative error $e=\left|\frac{S(\nu)^{2}-\sigma(\nu)^{2}}{\sigma(\nu)^{2}}\right|$ in the variance, the number of trials considered in the MC method should be:

$$
N=z_{\alpha / 2}^{2} \frac{\gamma(\nu)-1}{e^{2}}
$$

The results of applying Eq. (8) are given in Table 1 for different significance levels $\alpha$ and assumed relative errors $e$. In this case a population with 10000 trials was simulated to estimate $\gamma(\nu)$. The results reveal that this criterion is much more restrictive than that

\begin{tabular}{cccc}
\hline $\mathrm{e}$ & 0.05 & 0.02 & 0.01 \\
\hline 0.05 & 2331 & 3634 & 4663 \\
0.02 & 14571 & 22715 & 29146 \\
0.01 & 58282 & 90861 & 116582 \\
\hline
\end{tabular}

Table 1: Number of trials $N$, required to fulfil the variance coverage interval for different relative errors e and significance levels $\alpha$.

applied for the mean, since 12954 simulations are now needed to obtain $S(\nu)^{2}$ with a $3 \%$ error and $99 \%$ confidence level.

In order to validate the previous results, a second approach is used to quantify the accuracy of $S(\nu)^{2}$ which consists of following a classical bootstrapping strategy [28] in which no assumption of normality is required. This resampling technique is based on random sampling with replacement and can estimate the sampling distribution of almost any statistic (mean, variance, etc.) [29]. Starting from a large enough sample (of size $N$ ) of $\nu$, one can perform $N$ resamples $\left[\nu_{1}^{*}, \nu_{2}^{*}, \ldots, \nu_{N}^{*}\right]$, each one also of size $N$, taken from the original sample by using sampling with replacement. For each of these bootstrap samples we compute the desired statistic, in this case $\left[S\left(\nu^{*}\right)_{1}^{2}, S\left(\nu^{*}\right)_{2}^{2}, \ldots, S\left(\nu^{*}\right)_{N}^{2}\right]$, in order to obtain the histogram which provides an estimate of its PDF.

The principle of bootstrapping assumes that for large enough $N$, the bootstrap PDF of $S\left(\nu^{*}\right)^{2}$ tends to its real PDF. We start from three original initial samples of $n u$ with sizes $N=1000,5000$ and 10000. From these samples the histograms shown in Fig. 10 are built by the bootstrapping method. The $99 \%$ coverage intervals of these distributions are given 


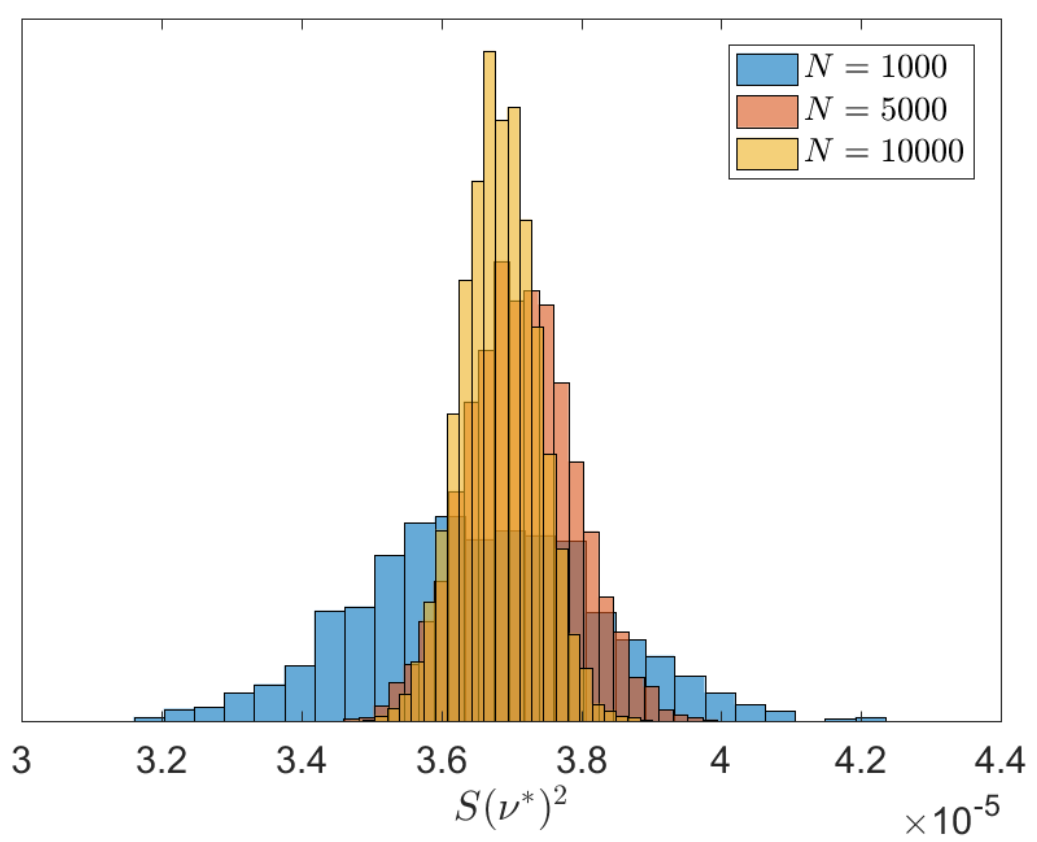

Figure 10: Bootstrap distributions of estimated variance $S\left(\nu^{*}\right)^{2}$, for samples of size $N=1000$, 5000 and 10000.

in Table 2, in which it can be seen that they become narrower with larger sample sizes. According to the results, the maximum relative error in the estimation is also displayed in the last column of Table 2 with a $99 \%$ certainty level.

\begin{tabular}{ccc}
\hline \multicolumn{3}{c}{$S\left(\nu^{*}\right)^{2}$} \\
\hline$N$ & $99 \%$ Cov. int. & $e_{\max }$ \\
\hline 1000 & $3.238 \cdot 10^{-3}-4.077 \cdot 10^{-3}$ & 11.55 \\
5000 & $3.524 \cdot 10^{-3}-3.912 \cdot 10^{-3}$ & 5.39 \\
10000 & $3.543 \cdot 10^{-3}-3.827 \cdot 10^{-3}$ & 3.94 \\
\hline
\end{tabular}

Table 2: $99 \%$ coverage interval and maximum error for the $S(\nu)$ bootstrap distribution.

In view of the results, if $\bar{\nu}$ is the quantity of interest, using $N=50$ trials, only about 0.002 of difference between $\bar{\nu}$ and the real $\mu(\nu)$ (less than $1 \%$ of relative error) is expected in $99 \%$ of the cases. Thus, we will use $N=50$ trials to perform MCS when looking for $\bar{\nu}$. However, if one is interested in the PDF of $\nu, N=10000$ trials could be employed in MCS to guarantee a certain accuracy in the obtained $S(\nu)^{2}$. In this case, the relative error between $S(\nu)^{2}$ and $\sigma(\nu)^{2}$ is limited by $3.94 \%$ at a $99 \%$ confidence level. If this result is compared with that obtained from the analytic expression (8), with $N=10000$ and 
$\alpha=0.01$, Eq. (8) gives a relative error of $3.41 \%$ which is in good agreement with the bootstrap estimated uncertainty for $S(\nu)^{2}$.

\section{Discussion of the numerical results}

As stated in Section 2, this work analyses the Benchmark catenary model [1], although the proposed procedure can be applied to any catenary topology. The model is composed of 20 spans but the results are taken only from the 10 central spans to avoid boundary effects. Following the recommendations in [17], the interaction force is low-pass filtered at $20 \mathrm{~Hz}$, so that the results are comparable to those obtained in [1].

The measurable output magnitudes for the Benchmark catenary are displayed in Table 3 and will be considered as reference values for comparison with those obtained for the actual installed catenaries with their corresponding installation errors.

\begin{tabular}{cccccc}
\hline$\nu$ & $t_{c l}(\%)$ & $\Delta z^{\max }(\mathrm{mm})$ & $F_{\text {inter }}^{\text {max }}(\mathrm{N})$ & $s_{s}^{\max }(\% 0)$ & $\Delta s_{s}^{\max }(\% 0)$ \\
\hline 0.2589 & 0 & 50.22 & 256.24 & 0 & 0 \\
\hline
\end{tabular}

Table 3: Measurable effects on the nominal Benchmark catenary when the train travels at $300 \mathrm{~km} / \mathrm{h}$.

The first examples are carried out at a train speed of $v=300 \mathrm{~km} / \mathrm{h}$ and an uplift force $F_{u p}=168.47 \mathrm{~N}$ is applied to the bottom mass of the pantograph model to fulfil the maximum mean contact force given in [23]:

$$
\mu\left(F_{\text {inter }}\right)=70+0.00097 v^{2}
$$

Whereas Section 6.1 gives a complete statistical quantification of the effects of installation errors on the static and dynamic features of the system, Section 6.2 only focuses on the mean values of these measurable effects. The next section is devoted to determining the influence of error variability when the train travels at different speeds. The influence of installation errors on the optimal initial sag of the contact wire (pre-sag) is also analysed. 


\subsection{Statistical quantification of the effects produced by instal- lation errors}

This section characterizes the effects of installation errors by computing their full PDFs; each source of uncertainty is first considered in isolation and then the most influential errors are analysed jointly.

The first error considered is that related to dropper lengths. The PDFs of the output parameters are given in Fig. 11, after performing $10000 \mathrm{MC}$ simulations. At first glance, a general deterioration of the system performance is clearly seen, since the mean of each variable is higher than the nominal deterministic values (solid lines in Fig. 11).
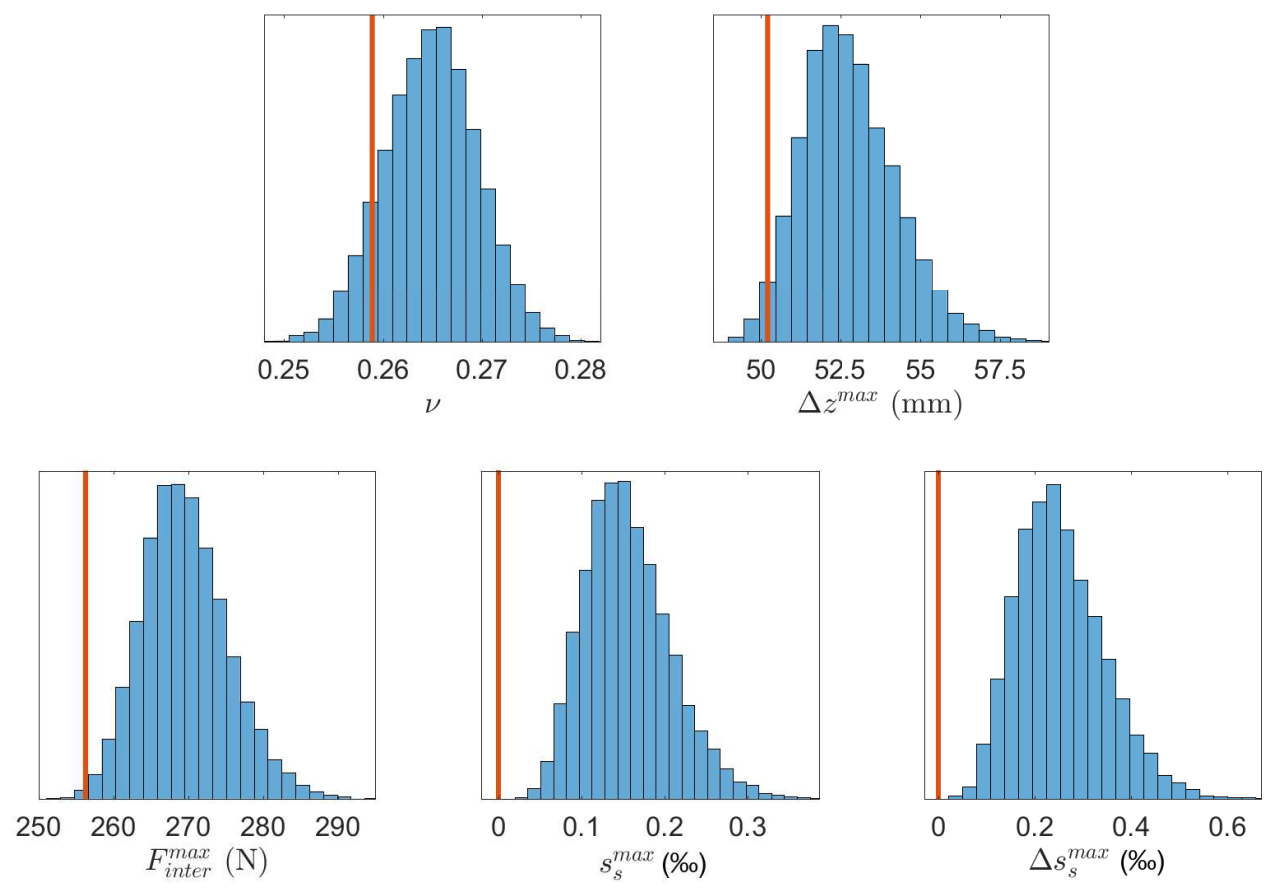

Figure 11: PDFs of output quantities for dropper length error.

The PDFs of $\Delta z^{\max }, F_{\text {inter }}^{\max }, s_{s}^{\max }$ and $\Delta s_{s}^{\max }$ present slightly positive skewness, while the opposite effect is found in the case of $\nu$. This installation error hardly produces any nondesirable contact losses between pantograph and catenary. If a contact loss is considered when the contact force before filtering is null in a single time-step ( $1 \mathrm{~ms}$ in this case), they only appear in 38 of the 10000 simulations performed, and in any case exceed $7 \mathrm{~ms}$ 
throughout a simulation.

If only deviations in dropper spacing are considered, the PDFs of the measurable effects plotted in Fig. 12 are obtained. In this case neither contact losses nor span slope changes can be noted. the PDFs of $\nu$ and $\Delta z^{\max }$ are around the value obtained with the nominal catenary. Its most important feature is its negligible influence on all the measured output quantities. On the basis of these results, we can conclude that this error barely affects the system dynamics and therefore will be disregarded hereinafter.
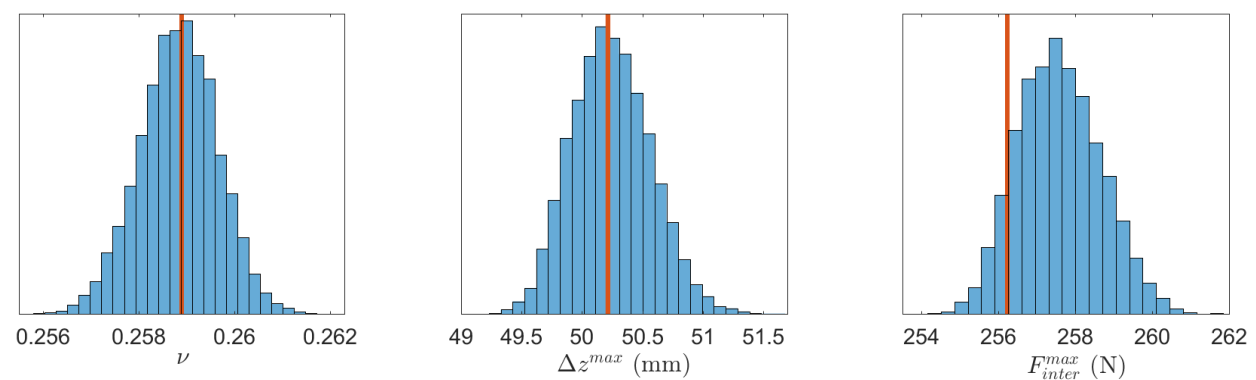

Figure 12: PDFs of output quantities for dropper spacing error.

The last error to be simulated concerns the height at which supports are placed. Considering that the same error affects both steady arm and messenger wire connections (3a and $3 \mathrm{~b}$ in Fig. 4) gives rise to the results shown in Fig. 13. Just as when considering dropper spacing error, the pantograph does not detach from the contact wire in any case, although this error has the biggest impact on the maximum span slope. The maximum registration arm uplift has also increased more than the values obtained from the simulated dropper length error, although $\nu$ is less influenced by support height errors than dropper length errors. In fact, almost half of the simulated trials have a beneficial effect on this magnitude.

In order to split the effects of height errors on messenger wire and steady arm connections to the bracket, we applied MCS allowing only for errors in the height of the placement of the links between steady arms and supports (3b error in Fig. 4). The results obtained show almost no dispersion, indicating that relative errors between the height of steady arm and messenger wire connections to the cantilever have a negligible effect on the considered output magnitudes. The hypothesis that the same error affects both steady arm and messenger wire connections is thus confirmed. 

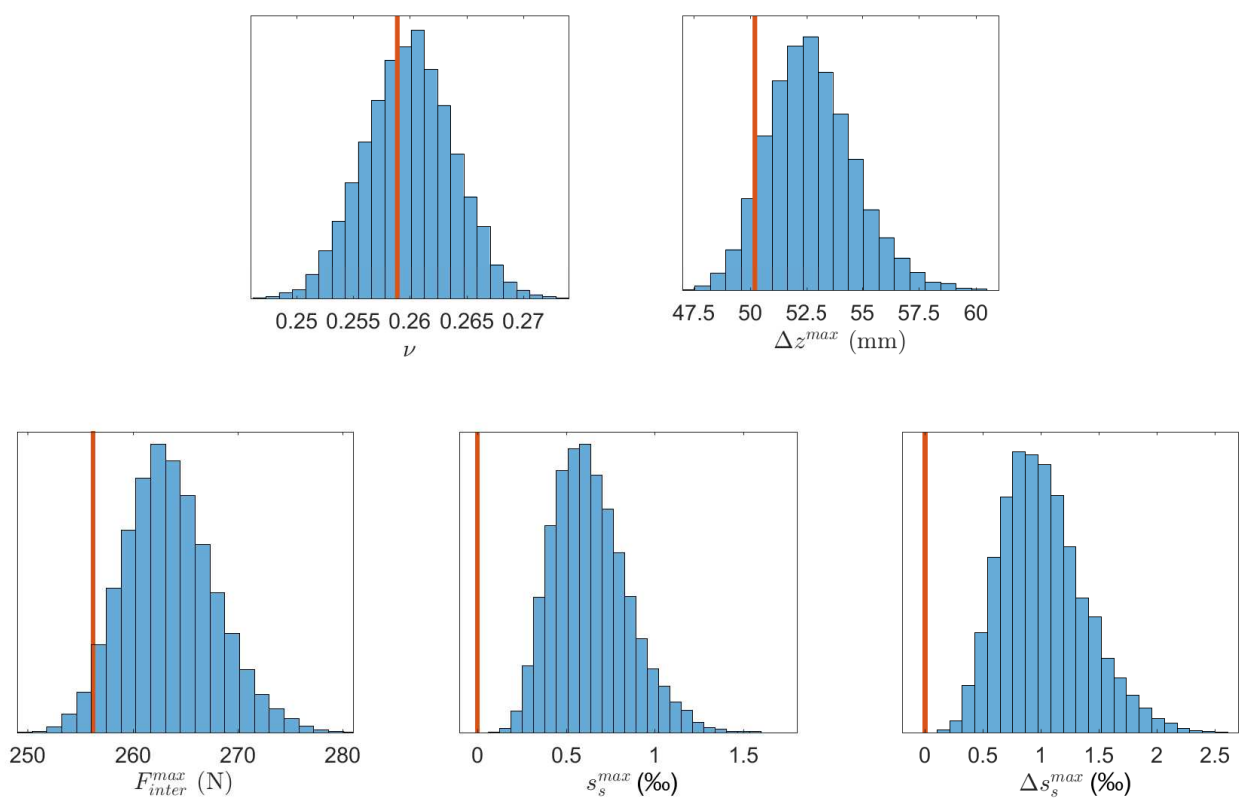

Figure 13: PDFs of output quantities for support height error.

Once each installation error has been analysed in isolation, we focus on the effects of the two most relevant errors occurring simultaneously, as can happen in practice. The results obtained are shown in Fig. 14, in which dropper spacing deviations are not considered due to their negligible influence on the measured magnitudes. In view of the PDFs obtained, $\nu$ deteriorates when both errors are simulated together. However, compared to the standalone results, there is only a slight tendency to worsen the behaviour of the system in the other magnitudes. It can also be seen that variability in $\nu$ and $F_{\text {inter }}^{\max }$ is mainly dominated by dropper length error, whilst variability in $\Delta z^{\max }, s_{s}^{\max }$ and $\Delta s_{s}^{\max }$ is dominated by errors in support height.

Focusing in greater detail on the coefficient of variation of the interaction force, $\nu$, the 95\% coverage interval, $\left[\begin{array}{ll}c_{\min } & c_{\max }\end{array}\right]$, is plotted in Fig. 15. In this figure the horizontal line represents the value of $\nu$ obtained with the nominal Benchmark catenary. The small variation of $\nu$ when dropper spacing error is considered can be clearly seen. The simulation of the support height error presents a wider $95 \%$ coverage interval, which is also centred to the nominal value. When dropper length error is allowed for, $\nu$ is more variable and most of the simulated trials show a higher value for this magnitude than the nominal one. This behaviour leads us to conclude that the dropper length error is the most harmful 

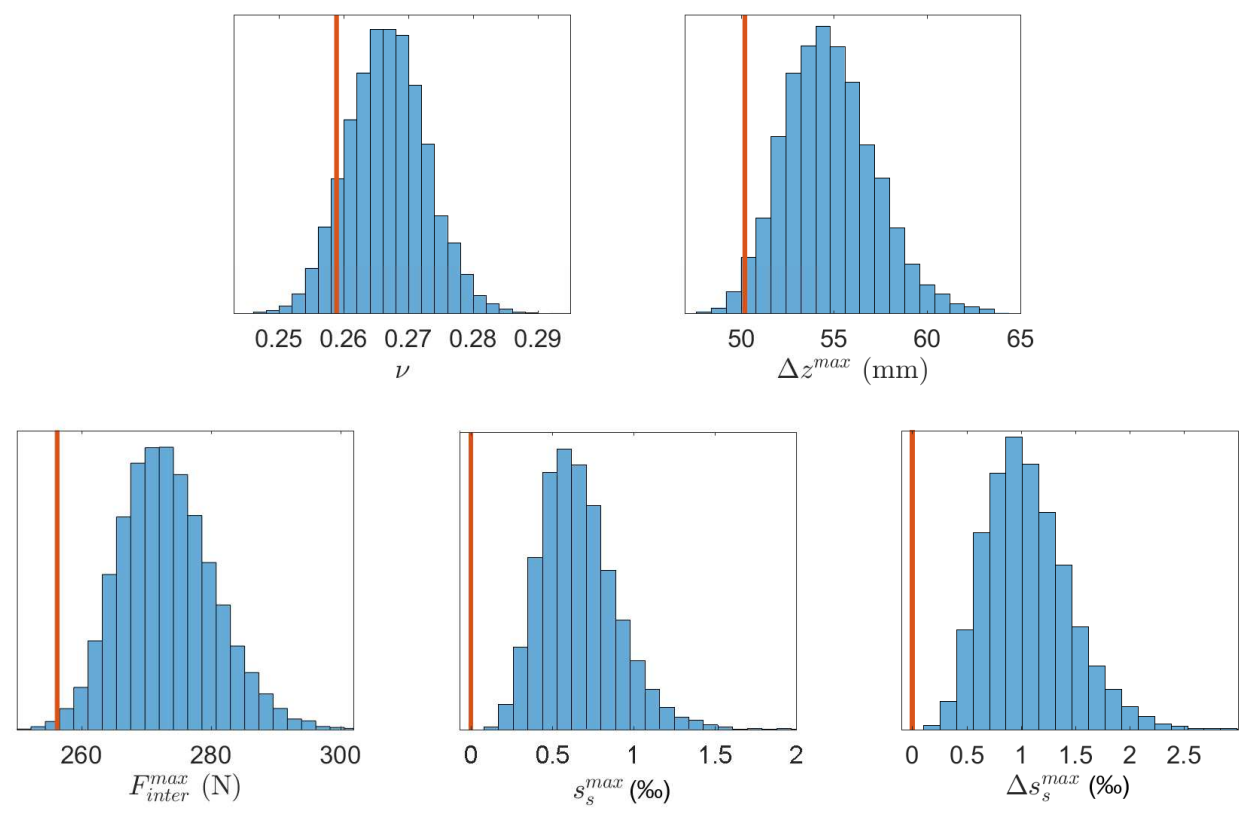

Figure 14: PDFs of output quantities allowing for both relevant installation errors, namely dropper length and support height errors.

error, and that great care should be given to this factor during installation. The worst scenario is found when dropper length and support height errors are simulated together; this synergy produces the widest $95 \%$ coverage interval and also the highest increase in the mean of $\nu$. Indeed, almost all the trials in the interval show worse current collection performance than the nominal catenary.

Table 4 summarises the most important results obtained for the PDF of $\nu$. The relative distance between the maximum and minimum edges of the $95 \%$ coverage interval,

$$
\Lambda=\frac{c_{\max }-c_{\min }}{c_{\min }} \cdot 100
$$

is also included as a quantifier of the variability in $\nu$ produced by each type of error. 


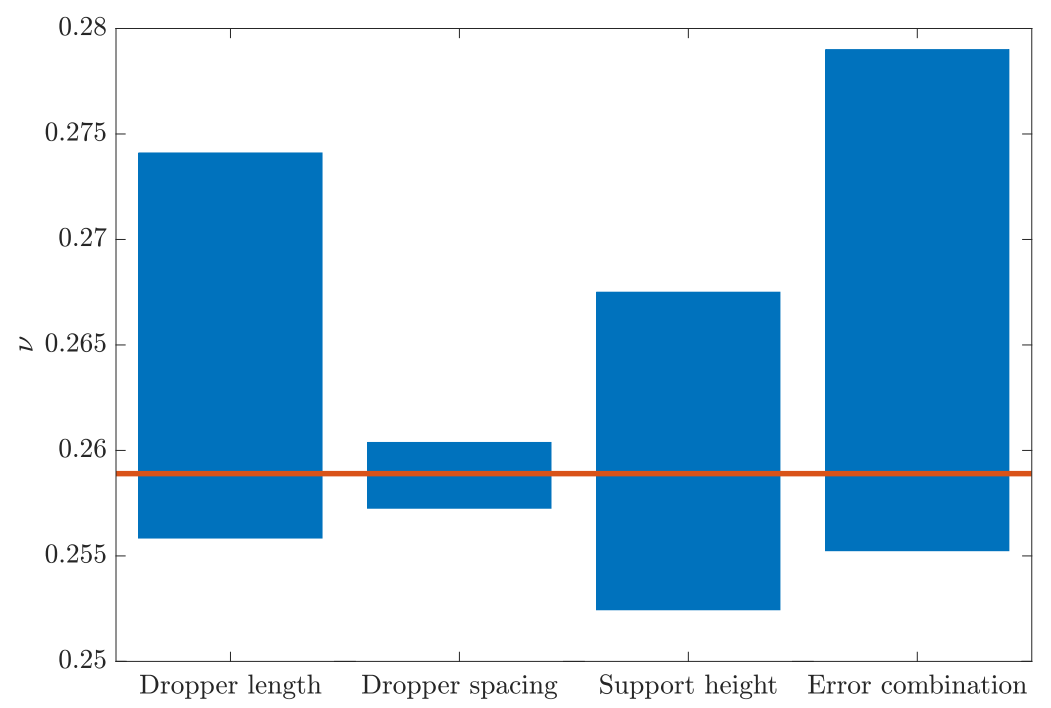

Figure 15: $95 \%$ coverage interval of $\nu$ for all the different studied scenarios.

\begin{tabular}{lcccc}
\hline Error type & $\bar{\nu}$ & $S(\nu)$ & $95 \%$ coverage interval & $\Lambda(\%)$ \\
\hline Dropper length & 0.2649 & $4.711 \cdot 10^{-3}$ & $0.2558-0.2741$ & 7.15 \\
Dropper spacing & 0.2588 & $8.138 \cdot 10^{-4}$ & $0.2572-0.2604$ & 1.24 \\
Support height & 0.2599 & $3.899 \cdot 10^{-3}$ & $0.2524-0.2675$ & 5.99 \\
Error combination & 0.2667 & $6.069 \cdot 10^{-3}$ & $0.2552-0.2790$ & 9.32 \\
\hline
\end{tabular}

Table 4: Summary of the statistical characterization of the PDF of $\nu$. Mean, standard deviation, 95\% coverage interval and $\Lambda$ ratio for the four studied scenarios.

\subsection{Mean evaluation of the effects produced by installation er- rors}

As already mentioned in Section 5.1, if only the mean value of a certain output magnitude is required instead of a full PDF description, only $50 \mathrm{MC}$ simulations need to be performed. With this number of simulations, the relative error expected in $\nu$ is less than $1 \%$, with a guaranteed confidence level of $99 \%$, although other output magnitudes may not reach this degree of accuracy.

The first row in Table 5 shows the mean values of the output magnitudes obtained from the PDFs in Fig. 14, which can be assumed to be the real population mean of each magnitude, since they are obtained from 10000 trials. The second row of the table gives the mean 


\begin{tabular}{cccccc}
\hline & $\nu$ & $\Delta z^{\max }(\mathrm{mm})$ & $F_{\text {inter }}^{\max }(\mathrm{N})$ & $s_{s}^{\max }(\%)$ & $\Delta s_{s}^{\max }(\% 0)$ \\
\hline$\mu$ & 0.2667 & 54.76 & 273.21 & 0.665 & 1.065 \\
$\tilde{\mu}$ & 0.2658 & 54.92 & 273.69 & 0.677 & 1.031 \\
Rel. Err. $(\%)$ & 0.34 & 0.29 & 0.18 & 1.80 & 3.19 \\
$\mu / \sigma$ & 43.94 & 22.29 & 37.53 & 2.77 & 2.67 \\
\hline
\end{tabular}

Table 5: Comparison of estimated mean values from a population of 50 individuals and those obtained from a population of 10000 individuals.

values obtained from only 50 simulations. The relative error of the latter estimation is given in the third row. It is clear that $\nu, \Delta z^{\max }$ and $F_{\text {inter }}^{\max }$ can be accurately estimated by this procedure, while estimating $s_{s}^{\max }$ and $\Delta s_{s}^{\max }$ would need a larger number of trials by the MC method. This can be explained by the ratio between the mean and the standard deviation of the PDF of each magnitude. The greater this ratio, the more accurate $\tilde{\nu}$ tends to be for a given number of trials.

\subsection{Influence of installation errors at different train speeds}

A nominal train velocity of $300 \mathrm{~km} / \mathrm{h}$ was considered in the examples discussed so far. However, any change in this parameter would affect the effects of the installation errors in the system dynamics. To study this phenomenon, the MC method was executed with 10000 trials, considering different train speeds, applying the appropriate uplift force and keeping all the other parameters constant. The simulations allow for both dropper length and support height errors.

The $95 \%$ coverage interval of $\nu$ is given in Fig. 16 for $v=200,250$ and $300 \mathrm{~km} / \mathrm{h}$. The uplift forces applied to the pantograph are $F_{u p}=114.07,138.33$ and $168.47 \mathrm{~N}$, respectively, to fulfil the maximum mean contact force requirement of Eq. (9). The horizontal lines denote the nominal $\nu$ value at each speed. $\nu$ clearly tends to decrease at lower train velocities. Regarding uncertainty, installation errors seem to be more detrimental at high velocities (250 and $300 \mathrm{~km} / \mathrm{h}$ ) since the $95 \%$ coverage interval is entirely located above its respective nominal $\nu$, despite being narrower. This deterioration in current collection quality cannot be appreciated at $v=200 \mathrm{~km} / \mathrm{h}$, because most of the simulated trials have a $\nu$ value lower than the nominal.

These results indicate that careful attention should be paid to accuracy when installing 


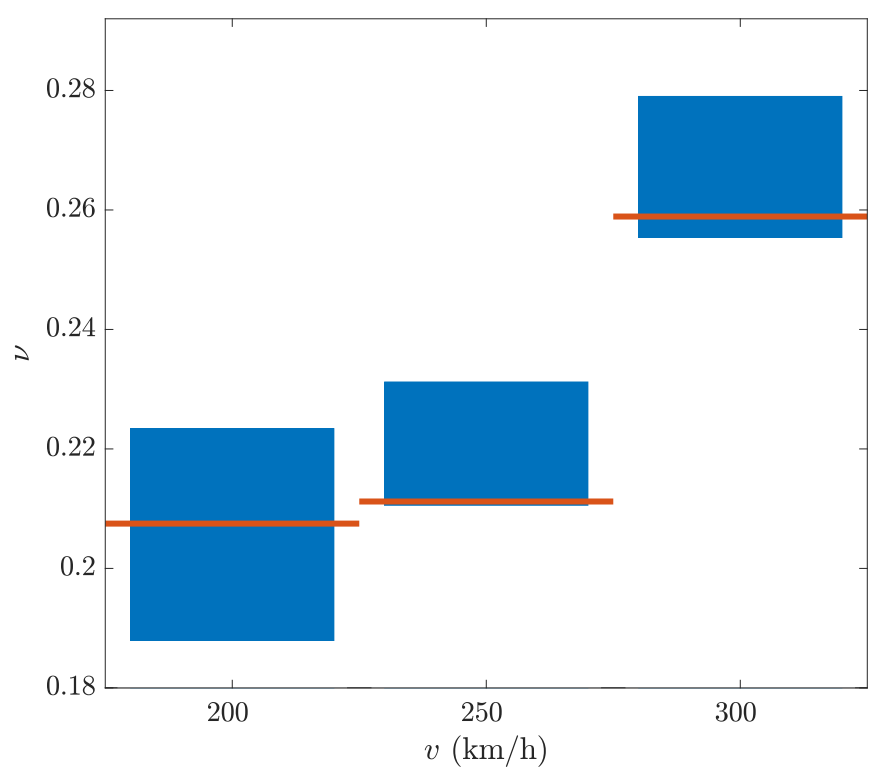

Figure 16: $95 \%$ coverage interval of $\nu$ at different train speeds including both dropper length and support height errors.

high-speed catenaries, since any errors tend to have an increasingly negative effect.

\subsection{Optimal robust pre-sag}

A certain amount of pre-sag is beneficial for current collection quality, especially in the medium speed range [30]. Optimal pre-sag values for a given train velocity are reported in [3], but only for deterministic simulations that ignored the variability of installation errors. Here we obtained a robust optimal pre-sag value, allowing for the uncertainty present in the installed catenaries.

Fig. 17 shows a comparison between $\bar{\nu}$ computed as the mean value of 50 samples with random installation errors (circles), and $\nu$ obtained from the nominal configuration (squares) when different pre-sag values are assigned. The study was carried out at three different speeds: 200, 250 and $300 \mathrm{~km} / \mathrm{h}$. The obtained optimal pre-sag is highlighted for each case with thick crosses in Fig. 17.

The first conclusion to be drawn from these results is the importance of considering the variability introduced by installation errors when seeking optimal catenary configurations. 

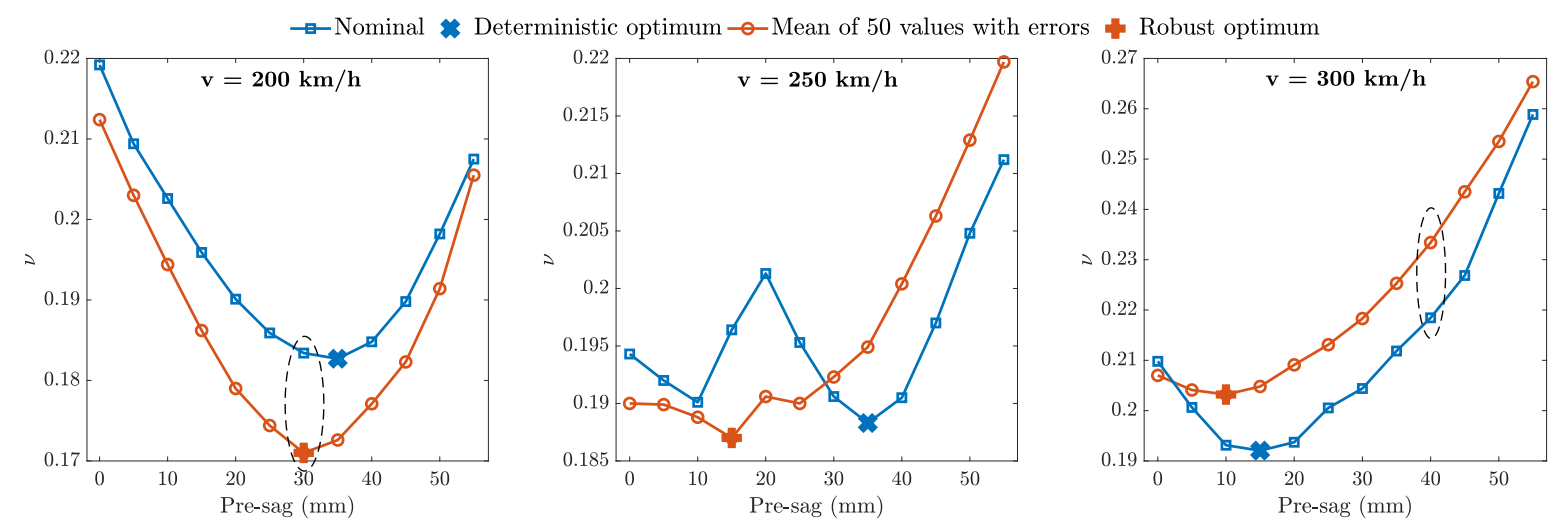

Figure 17: Comparison of the nominal $\nu$ and $\bar{\nu}$ computed from fifty trials with installation errors for catenaries with different initial sag values: $v=200 \mathrm{~km} / \mathrm{h}$ (left), $v=250 \mathrm{~km} / \mathrm{h}$ (centre) and $v=300 \mathrm{~km} / \mathrm{h}$ (right).

In this scenario, the optimal robust pre-sag differs from that obtained in the deterministic case.

Another interesting feature in Fig. 17 is that there are some catenary configurations in which $\bar{\nu}<\nu$, indicating that they behave better, on average, than in their corresponding nominal configuration. This unexpected behaviour occurs at low speeds $(200 \mathrm{~km} / \mathrm{h})$ in all the cases studied and at medium speeds $(250 \mathrm{~km} / \mathrm{h})$ in catenaries with low pre-sag $(<30 \mathrm{~mm})$.

In order to explain this phenomenon, we focus on two catenaries with opposing behaviour: one with $40 \mathrm{~mm}$ of pre-sag at $300 \mathrm{~km} / \mathrm{h}$ and another with $30 \mathrm{~mm}$ of pre-sag at 200 $\mathrm{km} / \mathrm{h}$ (points surrounded by dashed lines in Fig. 17). The Fourier transform of the lowpass filtered interaction force for both the nominal cases (squares) and the mean of fifty configurations with installation errors (circles) can be seen in Fig. 18.

If $\Xi=[0, \ldots, 20] \mathrm{Hz}$, we define $h=\left[h_{1}, h_{2}, \ldots, h_{n_{h}}\right]$ as the set of the harmonics of the fundamental span-pass frequency, and $\hat{h}=\Xi-h$ is therefore the set containing the remaining frequencies. In Fig. 18 most of the frequency content of $F_{\text {inter }}$ is located on $h$. The mean effect of installation errors seems to reduce the amplitudes of the $h$ frequencies in exchange for an increase in the frequency content of $\hat{h}$ due to the elimination of periodicity in contact wire height.

In order to quantify these effects, we can decompose $\nu$ into the contribution of both $h$ frequencies, $\nu_{h}$, and the remaining frequencies, $\nu_{\hat{h}}$, so that $\nu^{2}=\nu_{h}^{2}+\nu_{\hat{h}}^{2}$. Fig. 19 shows the 

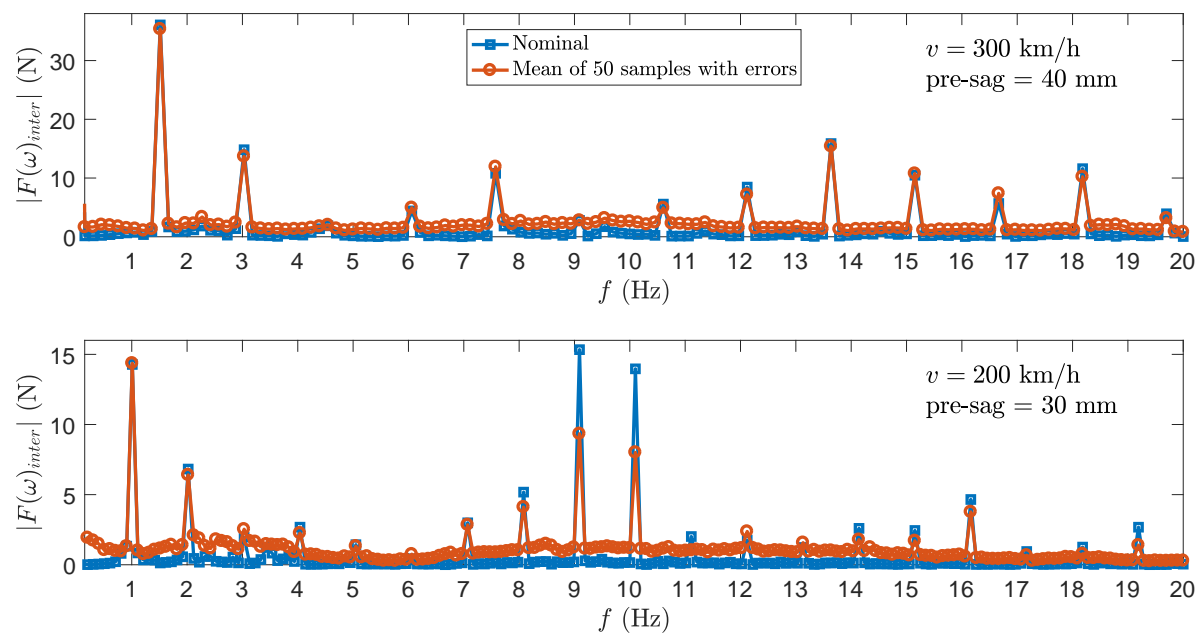

Figure 18: Frequency content of $F_{\text {inter }}$ for catenaries with $40 \mathrm{~mm}$ (top) and $30 \mathrm{~mm}$ (bottom) of pre-sag, considering nominal and actual installed scenarios with pantograph travelling at $v=$ $300 \mathrm{~km} / \mathrm{h}$ and $v=200 \mathrm{~km} / \mathrm{h}$, respectively.

difference $\bar{\nu}^{2}-\nu^{2}$ for both frequency set contributions: $h$ and $\hat{h}$. The increase seen in $\nu_{\hat{h}}$ seems to be more or less constant with respect to train speed and pre-sag, although the reduction of $\nu_{h}$ is clearly greater at lower speeds, with the maximum at $v=200 \mathrm{~km} / \mathrm{h}$.
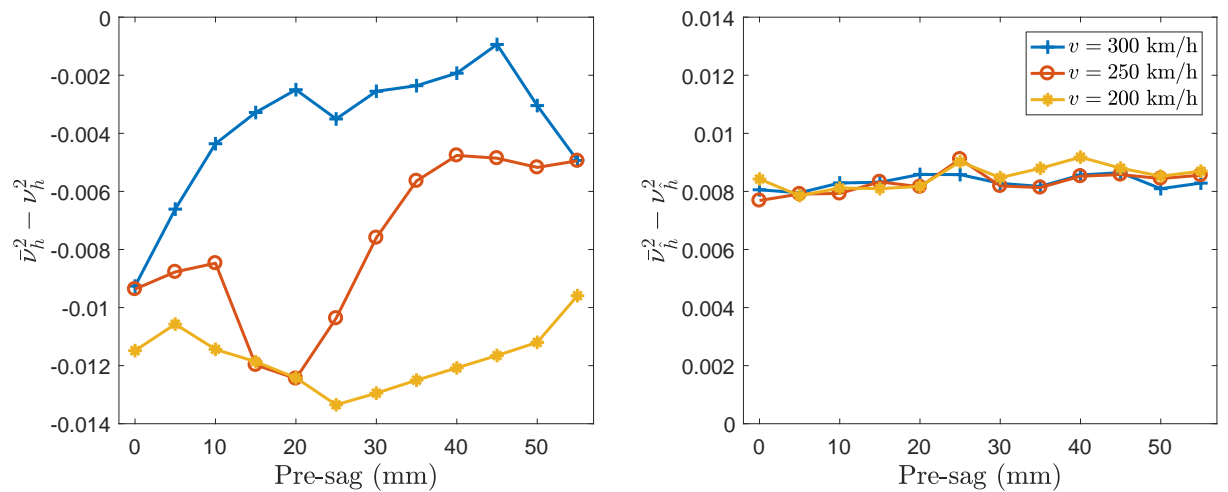

Figure 19: Difference between $\bar{\nu}^{2}$ and $\nu^{2}$ computed with the span-pass harmonics $h$ (left) or with the rest of the frequency content $\hat{h}$ (right).

To sum up, if the decrease in $\nu_{h}$ overcomes the increase in $\nu_{\hat{h}}$, the catenary in which allowance is made for installation errors, on average, behaves slightly better than the nominal catenary. In the case studied, this happens at low train speeds and low pre-sag values. 


\section{Conclusions}

This work proposes a method of simulating pantograph-catenary dynamic interaction that takes into account the variability introduced by catenary installation errors.

Three different error types were considered: those related to dropper length, dropper spacing and support height. After their statistical characterization, the Monte Carlo method was applied to propagate the uncertainty to the desired output quantities. A statistical study was then performed to obtain the number of $\mathrm{MC}$ trials required to control the accuracy of the PDF of the output quantities.

Allowing for these installation errors has a remarkable effect on the static and dynamic features of catenary behaviour. Current collection quality is clearly worse when dropper length and support height errors are simulated, whereas the spacing error has only minor effects. The results of the simulations carried out in this work indicate that the higher the speed, the more negative the effects of installation errors on current collection performance.

Another important conclusion is related to the optimal amount of pre-sag; this quantity not only varies with train speed but is also clearly affected when installation errors are taken into account in the simulations.

To summarise, all the results of this study highlight that considering the uncertainty due to catenary installation can affect the catenary dynamic behaviour.

\section{Acknowledgements}

The authors would like to acknowledge the financial support received from the FPU program offered by the Spanish Ministry of Education, Culture and Sports (MECD) under Grant Number FPU13/04191. The funding provided by the Regional Government of Valencia (PROMETEO/2016/007) and the Spanish Ministry of Economy, Industry and Competitiveness (TRA2017-84736-R) is also acknowledged. 


\section{References}

[1] S. Bruni, J. Ambrosio, A. Carnicero, Y. H. Cho, L. Finner, M. Ikeda, S. Y. Kwon, J. P. Massat, S. Stichel, and M. Tur, "The results of the pantograph-catenary interaction benchmark," Vehicle System Dynamics, vol. 53, no. 3, pp. 412-435, 2015.

[2] N. Zhou and W. Zhang, "Investigation on dynamic performance and parameter optimization design of pantograph and catenary system," Finite Elements in Analysis and Design, vol. 47, no. 3, pp. 288-295, 2011.

[3] S. Gregori, M. Tur, E. Nadal, and F. J. Fuenmayor, "An approach to geometric optimisation of railway catenaries," Vehicle System Dynamics, pp. 1-25, 2017.

[4] J. W. Kim, H. C. Chae, B. S. Park, S. Y. Lee, C. S. Han, and J. H. Jang, "State sensitivity analysis of the pantograph system for a high-speed rail vehicle considering span length and static uplift force," Journal of Sound and Vibration, vol. 303, no. 3, pp. 405-427, 2007.

[5] A. Collina, F. Fossati, and F. Resta, "An innovative OHL diagnosis procedure based on the pantograph dynamics measurements," in World Congress on Railway Research, Koln, 2001.

[6] A. Collina, F. Fossati, M. Papi, and F. Resta, "Impact of overhead line irregularity on current collection and diagnostics based on the measurement of pantograph dynamics," Proceedings of the Institution of Mechanical Engineers, Part F: Journal of Rail and Rapid Transit, vol. 221, no. 4, pp. 547-559, 2007.

[7] M. Aboshi and M. Tsunemoto, "Installation guidelines for shinkansen high speed overhead contact lines," Quarterly Report of RTRI, vol. 52, no. 4, pp. 230-236, 2011.

[8] O. Vo Van, J. P. Massat, C. Laurent, and E. Balmes, "Introduction of variability into pantograph-catenary dynamic simulations," Vehicle System Dynamics, vol. 52, no. 10, pp. 1254-1269, 2014.

[9] O. Vo Van, E. Balmes, and J. Massat, "Statistical identification of geometric parameters for high speed train catenary," in International Conference on Noise and Vibration, pp. 2-11. 
[10] N. Metropolis and S. Ulam, "The Monte Carlo method," Journal of the American Statistical Association, vol. 44, no. 247, pp. 335-341, 1949.

[11] S. Gregori, M. Tur, E. Nadal, J. Aguado, F. Fuenmayor, and F. Chinesta, "Fast simulation of the pantograph-catenary dynamic interaction," Finite Elements in Analysis and Design, vol. 129, pp. 1-13, 2017.

[12] M. Arnold and B. Simeon, "Pantograph and catenary dynamics: A benchmark problem and its numerical solution," Applied Numerical Mathematics, vol. 34, no. 4, pp. 345-362, 2000.

[13] A. Collina and S. Bruni, "Numerical simulation of pantograph-overhead equipment interaction," Vehicle System Dynamics, vol. 38, no. 4, pp. 261-291, 2002.

[14] M. Tur, L. Baeza, F. Fuenmayor, and E. García, "PACDIN statement of methods," Vehicle System Dynamics, vol. 53, no. 3, pp. 402-411, 2015.

[15] A. Shabana, "Computer implementation of the absolute nodal coordinate formulation for flexible multibody dynamics," Nonlinear Dynamics, vol. 16, pp. 293-306, 1998.

[16] J. Ambrosio, F. Rauter, J. Pombo, and M. Pereira, "A flexible multibody pantograph model for the analysis of the catenary-pantograph contact," vol. 23, pp. 1-27, 2011.

[17] EN 50318, "Railway applications. Current collection systems. Validation of simulation of the dynamic interaction between pantograph and overhead contact line," European Committee for Electrotechnical Standarization, 2002.

[18] M. Tur, E. García, L. Baeza, and F. Fuenmayor, "A 3D absolute nodal coordinate finite element model to compute the initial configuration of a railway catenary," Engineering Structures, vol. 71, pp. 234-243, 2014.

[19] N. M. Newmark, "A method of computation for structural dynamics," Journal of the Engineering Mechanics Division, vol. 85, no. 3, pp. 67-94, 1959.

[20] J. Ambrósio, J. Pombo, P. Antunes, and M. Pereira, "PantoCat statement of methods," Vehicle System Dynamics, vol. 53, no. 3, pp. 314-328, 2015.

[21] O. Vo Van, E. Balmes, A. Capitaine, and X. Lorang, "Sensitivity analysis of catenary geometry on current collection quality," The Third International Conference on 
Railway Technology: Research, Development and Maintenance, Cagliari, Sardinia, Italy, 2016.

[22] ENE TSI, "Technical specifications for interoperability relating to the 'energy' subsystem of the rail system in the union," European Commission, 1301/2014.

[23] EN 50367, "Railway applications. Current collection systems. Technical criteria for the interaction between pantograph and overhead line," European Committee for Electrotechnical Standarization, 2012.

[24] M. Á. Herrador, A. G. Asuero, and A. G. González, "Estimation of the uncertainty of indirect measurements from the propagation of distributions by using the MonteCarlo method: An overview," Chemometrics and Intelligent Laboratory Systems, vol. 79, no. 1, pp. 115-122, 2005.

[25] R. M. Dudley, "Central limit theorems for empirical measures," The Annals of Probability, pp. 899-929, 1978.

[26] D. G. Bonett, "Approximate confidence interval for standard deviation of non-normal distributions," Computational Statistics $\&$ Data Analysis, vol. 50, no. 3, pp. 775-782, 2006.

[27] S. Arnold, Mathematical Statistics. Prentice-Hall, 1990.

[28] B. Efron, "Bootstrap methods: another look at the jackknife," in Breakthroughs in statistics, pp. 569-593, Springer, 1992.

[29] B. Efron and R. J. Tibshirani, An introduction to the bootstrap. CRC press, 1994.

[30] Y. H. Cho, K. Lee, Y. Park, B. Kang, and K. N. Kim, "Influence of contact wire pre-sag on the dynamics of pantograph-railway catenary," International Journal of Mechanical Sciences, vol. 52, no. 11, pp. 1471-1490, 2010. 